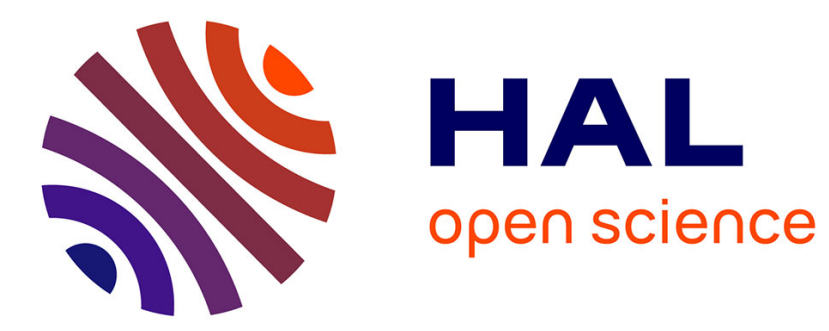

\title{
A Recursive Estimation Algorithm to Track Aircraft Model Parameters
}

\author{
Georges Hardier, Gilles Ferreres, Cédric Seren
}

\section{To cite this version:}

Georges Hardier, Gilles Ferreres, Cédric Seren. A Recursive Estimation Algorithm to Track Aircraft Model Parameters. IEEE SysTol'16, Sep 2016, BARCELONE, Spain. hal-01404475

\section{HAL Id: hal-01404475 \\ https://hal.science/hal-01404475}

Submitted on 28 Nov 2016

HAL is a multi-disciplinary open access archive for the deposit and dissemination of scientific research documents, whether they are published or not. The documents may come from teaching and research institutions in France or abroad, or from public or private research centers.
L'archive ouverte pluridisciplinaire HAL, est destinée au dépôt et à la diffusion de documents scientifiques de niveau recherche, publiés ou non, émanant des établissements d'enseignement et de recherche français ou étrangers, des laboratoires publics ou privés. 


\title{
A Recursive Estimation Algorithm to Track Aircraft Model Parameters
}

\author{
G. Hardier, G. Ferreres, and C. Seren
}

\begin{abstract}
On-line parameter tracking is often an attractive option for advanced FDD/FTC systems that require a global monitoring in contrast with a local (component) one. This is the case for impaired aircraft, but also for under-equipped vehicles (UAVs, small airplanes), since an updated model is a prerequisite for many indirect adaptive or reconfigurable control algorithms. Many schemes have been proposed since the $80 \mathrm{~s}$ to deal with the tricky problem of on-line estimation. However for aircraft, their computational burden is often incompatible with the constraints resulting from onboard implementation and certification issues. As a result, this paper relies on the well-known pros of frequency domain techniques, but proposes a fully recursive algorithm simple enough to comply with those requirements. The approach is applied to the parameter tracking of a civil aircraft on view of designing an adaptive gain-scheduled flight control law.
\end{abstract}

\section{INTRODUCTION}

This paper deals with the monitoring aspects related to FDD/FTC, and more precisely with the possibility to update the system modeling onboard, via a continuous estimation of its parameters. Actually, online tracking in near-real time is often the only alternative for some advanced FDD/FTC systems [13,23,31] that require a global (system-wide) monitoring of the system in contrast with a local (component) level monitoring. An example for aircraft is the detection of icing (de-icing off or faulty), in contrast with actuator failures that can be sensed by servo mechanisms simply by comparing control orders with surface deflections when measured. But a loss of actuator efficiency resulting from airframe damages might be also undetected by electrical/mechanical subsystems, and a global monitoring can also be beneficial for underequipped vehicles (UAVs, small airplanes).

More generally, parameter estimation, also called Parameter IDentification (PID), refers to the task of updating the parameters of a mathematical model from i/o data. For aircraft, a batch or off-line version of PID is generally useful to adjust the prior initial modeling issued from CFD computations or wind tunnel testing. However, in abnormal conditions resulting from any impairment (fault, damage, etc.), that modeling can become unsuited again, and thus requires on-line adaptation, especially for FTC purposes since an updated model is a prerequisite for many indirect adaptive or reconfigurable control algorithms [4,27,28]. When a post-fault model is available with an updated set of parameters, the Fault Accommodation process can be completed by scheduling or redesigning the control laws $[3,7,10]$.

The approach presented in this paper is part of a FTC scheme based on indirect adaptive control whose principle is to estimate some of the model parameters and to adjust a

G. Hardier, G. Ferreres and C. Seren are with ONERA/DCSD, Toulouse, France, [(+33)5 622527 77; Georges.Hardier@onera.fr].

This work was performed in the framework of the RECONFIGURE European Project: Grant agreement №: FP7-314544. controller on-line in terms of the updated estimates. Actually, gain-scheduling is classically used to control an airplane along a trajectory, the controller gains depending on measured or estimated flight parameters, such as airspeed, Mach number, mass and so on. However, in abnormal situations (actuator faults, icing), or if a scheduling parameter is unavailable due to a sensor fault (e.g., airspeed), adaptive control is an attractive solution to recover closed loop performance by scheduling the gains w.r.t. the aerodynamic model coefficients instead. This requires to obtain a priori guaranteed stability and performance properties of the adaptive closed loop, and to decrease the computational time and complexity to make onboard implementation possible. A detailed description of the associated FTC scheme is given in the companion paper [8].

Regarding PID, the main issue is to develop a method for estimating the model parameters on-line, with a low computational burden and the capability to track time-varying parameters despite measurement noises, poor data information content (in cruise condition when variables are likely to be almost constant during long periods), and external disturbances (turbulence). Whatever method used, residual errors can sometimes be large and a measure of the parameter accuracy should also be provided, so that some logic can be introduced: if the estimated scheduling parameters are not reliable enough, one can switch to a robust back-up controller ensuring minimal performance properties. Due to possible ill-conditioning, the method should also include some form of regularization $[22,29]$, although most on-line techniques are based on Equation Error (EE) minimizations [25] and involve only a few parameters in practical applications (e.g., via a tuneddown modeling). A contrario, Output Error methods [13] usually involve an extended set of parameters that are likely to reinforce the implementation issues. They are also less robust to the effect of unmodeled disturbances (turbulence).

Algorithms for recursive parameter estimation have been developed for real-time applications in various fields such as industrial processes, robotics and aerospace [14,15,22]. Most theoretical aspects were covered by early reference publications $[1,21]$. In aeronautics, the early attempts to benefit from on-line PID date back to the 80s, but they were limited by the available computational power of onboard processors. The common Time Domain (TD) methods are usually based on recursive/sequential Least-Squares (LS), or Extended Kalman Filters to cope with nonlinearities [5,12,20]. More recently, this challenge has also been addressed by recursive subspace identification of linear or LPV models. However, the relevance of such advanced multi-model or nonlinear schemes for on-board implementations is dubious due to the present limitations of on-board computers, especially for $\mathrm{A} / \mathrm{C}$ because of certification issues and code verification. To give an example, these limitations preclude the use of any iterative algorithm which would result in some unbounded 
computation loop through the data. Hence complex operations like non analytical matrix inversions should be banned. That is why we look in this paper for a simple enough approach, more likely to satisfy those real time constraints, and that could constitute a viable alternative for updating the models required by the most advanced FDD/FTC schemes.

Considering the implementation issues, TD algorithms could seem preferable at first sight, as the basic ones only involve very simple operations (e.g., by replacing matrix inversion with a scalar division). However, advanced strategies are usually required for regularizing the estimation process, and some of their advantages can thus be wasted (see [14] for an overview of the pros and cons of TD methods). By contrast, Frequency Domain (FD) techniques have many desirable features for on-line applications that other (TD) estimation methods do not have. The computation time is greatly reduced by processing only a limited amount of frequencies within the bandwidth of interest, and the resulting indirect filtering of the wide-band disturbances (e.g., LF mismatch and HF noise) improves the estimation accuracy $[11,13,18]$. The standard deviation of the estimation errors can also be evaluated without any additional cost. Furthermore, the availability of efficient tools making the transition from TD to FD possible, such as the recursive Fourier Transform (FT), greatly facilitates their use, and this was stressed in many publications during the last 20 years [2,11,18,24-26,30]. Fig. 1 gives a schematic representation of the way the FD information is linked to the PID process. Moreover, many issues specific to on-line PID are mitigated when using FD approaches instead of TD ones: estimates are nearly unbiased even in the presence of noisy $\mathrm{i} / \mathrm{o}$ data in case of collinearity in the regressors, the estimation errors do not require to be improved afterwards due to possible colored residuals, and so on (see [25] for all these practical aspects).

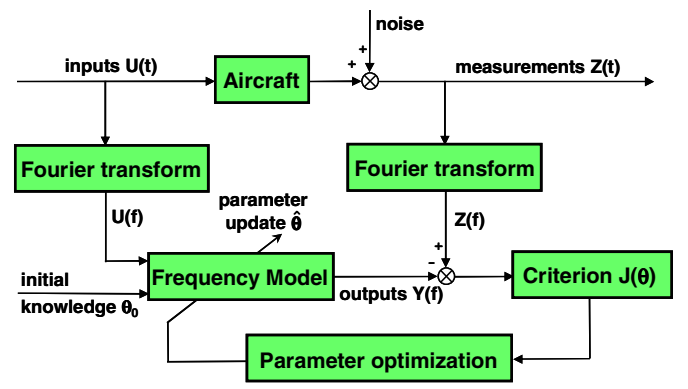

Fig. 1. The FD identification process

Otherwise, TD and FD estimation schemes can rely on a fully recursive algorithm (making use of measurements as soon as they are available), or alternatively on a kind of sequential procedure, processing moving data windows with a lower rate to get a succession of piecewise constant values [4,14]. In [13], a sequential estimation process was proposed for monitoring a civil aircraft, that permits pre and postprocessing stages to be included in the procedure to prevent from and to filter out inaccurate estimations. Hence, those 2 categories of methods have their own pros and cons [16], even if recursive schemes should be favored for on-line implementation. However, FD approaches are again more suitable to define hybrid recursive/sequential procedures because the information naturally accumulates in the signals' FT, which behave like storing memories and can be managed with more flexibility (truncation, forgetting, resetting).

Finally, one of the major reasons for which recursive TD algorithms are more popular than FD ones comes from some additional computation complexity, which has prevented from developing a fully recursive formulation of the FD approach, unlike TD case. This paper improves the state-ofthe-art existing approaches and proposes a recursive procedure from start to finish, permitting the practical implementation constraints to be satisfied, and the algorithm to be possibly embedded in aircraft computers. Other minor issues in the FT expressions of the signal derivatives are also properly settled, which are often disregarded for sequential processing thanks to the detrending achieved prior to FT computations, but which cannot as far as recursive algorithms are concerned. To sum up, the point is that FD approaches can also be simplified in order to reduce their computational complexity, similarly to what is done for TD formulations, and they can also include similar advanced mechanisms like data forgetting $[14,21]$. To cover these aspects, §II outlines the characteristics of the proposed recursive FD method. §III depicts the performances achieved in realistic flight conditions using the industrial non linear simulator of the RECONFIGURE project. §IV draws conclusions and gives some prospects.

\section{II.PRINCIPLES OF THE FREQUENCY DOMAIN PID}

\section{A. From time domain to frequency domain}

The transition to the FD is classically realized by means of the standard FT of the TD signals. As these ones are only available over a limited period of time $[0, T]$, the finite FT is used instead, which leads to the following relations for a signal $x(t)$, its time derivative $\dot{x}(t)$, and a constant value $b$ (associated to a bias for instance):

$$
\begin{cases}x(t) & \Leftrightarrow F_{\mathrm{T}}[x(t)]=X(\omega, T)=\int_{0}^{T} x(t) e^{-j \omega t} d t \\ b & \Leftrightarrow F_{\mathrm{T}}[b]=b\left(1-e^{-j \omega T}\right) / j \omega \\ \dot{x}(t) & \Leftrightarrow \mathcal{F}_{\mathrm{T}}[\dot{x}(t)]=j \omega X(\omega, T)+x(T) e^{-j \omega T}-x(0)\end{cases}
$$

Practically, from the sampled values of $x(t)$, the finite FT can be computed via a rectangular numerical integration:

$X(\omega, T) \approx \Delta t \sum_{n=0}^{N-1} x(n \Delta t) e^{-j \omega n \Delta t}=\Delta t \tilde{X}(\omega)$

using $N$ values equally spaced over $[0, T]$, with a sampling period $\Delta t$ (see for instance [18]). The term $\tilde{X}(\omega)$ represents the Discrete Fourier Transform (DFT) of the samples $\{x(n \Delta t), n=0, \ldots, N-1\}$. Efficient tools are available for computing this quantity, namely the Fast Fourier Transform (FFT) and the Chirp z-transform (CZT). The latter permits a desired frequency resolution to be chosen independently of the length of the time interval used, but is less effective as far as the computation time is considered. If we assume that the DFT is computed via standard FFT, from $N$ data samples equally spaced over the time interval $[0, T]$, this algorithm calculates $N$ values of the DFT over the frequency interval $[0,2 \pi N / T]$, equally spaced too with a step $\Delta \omega=2 \pi / T$.

More elaborated algorithms are available to reduce the inaccuracy resulting from (2), but these refinements are generally not useful when the sampling period is higher enough [24]. Otherwise, we will see in the sequel that the 
recursive algorithm can provide FT at any selected frequency without the extra computational cost of CZT.

\section{B. Standard formulation of a sequential algorithm}

From now, we will consider a general formulation of the problem, leading to other types of parameters being considered in addition to the stability and control derivatives. When applying PID techniques, it is generally advisable to estimate initial conditions and state/output biases in order to cope with i/o measurement offsets or model structure uncertainties. Consequently, the dynamics will be expressed in state-space form by the following linearized equation:

$$
\left\{\begin{array}{l}
\dot{x}(t)=A(\Theta) x(t)+B(\Theta) u(t)+b_{x} \\
y(t)=C(\Theta) x(t)+D(\Theta) u(t)+b_{y}
\end{array}\right.
$$

with the initial condition given by $x(0)=x_{0}$. Matrices $A, B, C, D$ include the stability and control derivatives $\Theta$ to be estimated, that are assumed to be constant or at least to vary slowly during the flight with respect to the PID process and updating rate. In the case of aircraft for instance, the states of vector $x$ are the airplane speeds and angular velocities in body axis, as well as Euler angles. The vector $u$ collects the surface deflections, whereas the measurement vector $y$ includes state components, air data angles and load factors. $b_{x}$ and $b_{y}$ represent the state and output biases.

Thanks to (1), if $\omega$ is chosen to be a multiple of the frequency step $\Delta \omega$. (which happens for instance when using the FFT), by omitting the dependencies on $\Theta$ and by denoting $X \equiv X(\omega), U \equiv U(\omega), Y \equiv Y(\omega)$, (3) becomes [13]:

$$
\left\{\begin{aligned}
j \omega X & =A X+B U+b_{x} T \delta(\omega)+x(0)-x(T) \\
Y & =C X+D U+b_{y} T \delta(\omega)
\end{aligned}\right.
$$

where $\delta(\omega)$ denotes a Dirac function in the FD, such that $\delta(\omega)=1$ for $\omega=0$, and $\delta(\omega)=0$ for $\omega=2 k \pi / T \neq 0$ (since $\left(1-e^{-j \omega T}\right) / j \omega=T$ for $k=0$, and 0 for $\left.k \neq 0\right)$.

As a result, the initial and final conditions $x(0), x(T)$ are translated into a bias that impacts on all frequencies: localized effects in the TD are translated into broadband effects in the FD, and vice versa. A contrario, the biases act as broadband inputs in the TD but just modify the zero frequency. To get the most out of these specificities, it is generally worthwhile to discard the zero frequency during the identification stage, which avoids the state and output biases having to be estimated. Thus, (4) is further simplified and reduces to:

$$
\left\{\begin{aligned}
j \omega X & =A X+B U+x(0)-x(T) \\
Y & =C X+D U
\end{aligned}\right.
$$

Hence, the discrepancies between the initial and final conditions of the states should be estimated in addition to the other parameters $\Theta$, if not zero. However, this issue has not been paid much attention and is often ignored in practice $[13,19]$, which could be only justified in a pinch if a suitable preprocessing is inserted in a sequential algorithm. Otherwise, the vector of parameters should be conveniently extended to include the $\operatorname{dim}(x)$ corresponding values. If we also assume that all the state components are measured, in addition to the i/o ones, an EE approach can be used, that consists in minimizing a set of cost functions [24-26]:

$$
J_{i}(\Theta)=\frac{1}{2} \sum_{k=1}^{M}\left|j \omega_{k} x_{i}\left(\omega_{k}\right)-A_{i} X\left(\omega_{k}\right)-B_{i} U\left(\omega_{k}\right)-b_{i}\right|^{2}
$$

where the subscript $i$ corresponds to the $i^{\text {th }}$ state equation, and $\omega_{k}$ to one of the $M$ frequencies available from the TD to FD transformation $(M \leq N) . A_{i}, B_{i}$ are the $i^{\text {th }}$ rows of matrices $A$ and $B$, whereas $x_{i}\left(\omega_{k}\right)$ is the $i^{\text {th }}$ component of the vector $X\left(\omega_{k}\right)$ and $b_{i}=x_{i}(0)-x_{i}(T)$. The same kind of expression can also be established for each output equation, permitting the parameters included in matrices $C, D$ to be estimated.

By collecting the different frequencies in vector and matrix forms, to make the summation of (6) disappear and by denoting $\Xi$ the extended vector of unknown parameters in $A_{i}, B_{i}$ and $b_{i}$, the cost (6) turns to the LS criterion [26]:

$$
\begin{aligned}
& J_{i}(\Xi)=\frac{1}{2}(\mathcal{Y}-X \Xi)^{\dagger}(\mathcal{Y}-X \Xi)+\frac{1}{2}\left(\Xi-\Xi_{0}\right)^{T} \Sigma_{0}^{-1}\left(\Xi-\Xi_{0}\right) \\
& \text { with : }\left\{\begin{array}{l}
X^{T}=\left[\begin{array}{ccc}
X\left(\omega_{1}\right) & \ldots & X\left(\omega_{M}\right) \\
U\left(\omega_{1}\right) & \ldots & U\left(\omega_{M}\right) \\
1 & \ldots & 1
\end{array}\right] \\
\Upsilon^{T}=\left[\begin{array}{llll}
j \omega_{1} x_{i}\left(\omega_{1}\right) & \ldots & j \omega_{M} x_{i}\left(\omega_{M}\right)
\end{array}\right]
\end{array}\right.
\end{aligned}
$$

where $\dagger$ is the complex conjugate transpose operator. In (7), the conditioning of the resulting optimization has been possibly improved by introducing some a priori knowledge about the expected value of the parameters. This form of regularization can be especially useful for weakly identifiable parameters, by softening their variations and improving the convergence. The principle of this Bayes-like estimation consists in adding a penalty term to the LS criterion to weight the $\Xi$ increments w.r.t. prior values $\Xi_{0}$ through a corresponding covariance $\Sigma_{0}$ (usually a diagonal matrix). (7) is nothing but a standard LS regression with complex data instead of real ones. The well-known solution to this problem and the estimated error covariance are given by $[18,26]$ :

$$
\left\{\begin{array}{l}
\hat{\Xi}=\left[R\left(X^{\dagger} X\right)+\Sigma_{0}^{-1}\right]^{-1}\left[R\left(X^{\dagger} X\right)+\Sigma_{0}^{-1} \Xi_{0}\right] \\
\operatorname{Cov}(\hat{\Xi})=\sigma^{2}\left[R\left(X^{\dagger} X\right)+\Sigma_{0}^{-1}\right]^{-1}
\end{array}\right.
$$

where $R()$ is the real part of a complex value. Practically, to compute the covariance matrix from the available data, especially the diagonal terms giving access to the parameter errors, the EE variance $\sigma^{2}$ is usually evaluated a posteriori from the residual errors, resulting in [26]:

$$
\hat{\sigma}^{2}=(\mathcal{X}-X \hat{\Xi})^{\dagger}(\mathcal{X}-X \hat{\Xi}) /[M-\operatorname{dim}(\Xi)]
$$

\section{Towards a fully recursive algorithm}

As regards practical aspects for on-board implementation, it should be noticed that the summation of (2) makes very simple operations possible, as mentioned for instance in [24]:

$$
\left\{\begin{array}{l}
\tilde{X}_{n}(\omega)=\tilde{X}_{n-1}(\omega)+x(n \Delta t) e^{-j \omega n \Delta t} \\
e^{-j \omega n \Delta t}=e^{-j \omega \Delta t} e^{-j \omega(n-1) \Delta t}
\end{array}\right.
$$

to evaluate the DFT at time $n \Delta t$ from its previous value at time $(n-1) \Delta t$. For a given frequency $\omega$, the quantity $\exp (-j \omega \Delta t)$ is constant, so that the updating of the DFT just requires two multiplications and one addition, resulting in a very low computational effort. As the estimation process is also likely to involve state derivatives, the measurements of which are usually not available, a recursive formulation similar to (10) can be worthwhile for signals $\dot{x}(t)$ to avoid a pseudo-derivation from the available signal $x(t)$, but also to 
avoid the extra $b_{i}$ to be estimated. The idea is to derive LS costs similar to (6), but involving $\dot{X}\left(\omega_{k}\right)$ directly in the computation of the error terms as this is the case for a measurement $Y\left(\omega_{k}\right)$. This idea is new and differ from the two usual ways consisting either in computing an estimate of $\dot{x}(t)$ in the TD or in replacing $\dot{X}(\omega)$ by $j \omega X(\omega)$ in the FD, which is incorrect whenever $b_{i} \neq 0$. From (1)-(2), we easily get:

$\dot{X}_{n}(\omega)=j \omega X_{n}(\omega)+x(n \Delta t) e^{-j \omega n \Delta t}-x(0)$

with $X_{n}(\omega) \approx \Delta t \tilde{X}_{n}(\omega)$. Hence, recursive expressions can be implemented (for any signal $x(t)$ even if it is not a state component consistent with the general formulation of $\S$ II.B), by denoting $X_{n} \equiv X_{n}(\omega), \dot{X}_{n} \equiv \dot{X}_{n}(\omega), x_{n} \equiv x(n \Delta t)$ :

$\left\{\begin{array}{l}\dot{X}_{n}-\dot{X}_{n-1}=j \omega\left(X_{n}-X_{n-1}\right)+\left(x_{n} e^{-j \omega \Delta t}-x_{n-1}\right) e^{-j \omega(n-1) \Delta t} \\ X_{n}-X_{n-1} \approx \Delta t\left(\tilde{X}_{n}-\tilde{X}_{n-1}\right)=\Delta t x_{n} e^{-j \omega n \Delta t}\end{array}\right.$

When using these coupled recursive updates for on-line purposes, older information can be overweighted regarding to recent ones, which can result in much delay in the adaptation process. That's why data forgetting can be used in conjunction with (10), to remove the effect of oldest data by working on a limited time window [26]. In that case, the choice of the window width results from a trade-off between the amount of information available from the data and the sensitivity of the detection method to $\mathrm{A} / \mathrm{C}$ parameter variations. This remark also applies to a pure sequential approach, where a non recursive form of FT is applied to the data [13], resulting in a succession of piecewise constant values, updated only after some seconds and hence delayed w.r.t. the varying parameters.

Whatever approach followed, a time history of the past data could be required to enable those calculations. To avoid such a storage, and to develop a recursive algorithm, it is still possible to benefit from the linearity of the FT w.r.t. the signals to implement a forgetting process similar to the one of TD exponential forgetting [14], and that simply involves multiplying the previous FT by a forgetting factor $\lambda(\lambda<1)$ [26]. Applying this trick to (12) yields (denoting $x_{n}^{*}=x_{n} e^{-j \omega \Delta t}$ ):

$\left\{\begin{array}{l}\dot{X}_{n} \approx \lambda \dot{X}_{n-1}+\left[(j \omega \Delta t+1) x_{n}^{*}-x_{n-1}\right] e^{-j \omega(n-1) \Delta t} \\ X_{n} \approx \lambda X_{n-1}+\Delta t x_{n}^{*} e^{-j \omega(n-1) \Delta t}\end{array}\right.$

We are now going to address a key feature of the proposed algorithm. With TD methods, the arrival of a new sample only results in the addition of a new row in the regression matrix [14]. So, the information matrix (or its inverse) can be updated via simple (and light) computations thanks to the Woodbury formula. With FD methods, a new sample contributes to modify all the $M$ rows of the matrix $X$ through the FT updates of (12). That is why the usual algorithms often rely on a sequential estimation of the parameters, due to the lack of a recursive update of the matrix $R=R\left(X^{\dagger} X\right)$ or of its inverse. However, a recursive version can still be derived, almost similar to the TD ones, by noticing that:

$\underline{X}_{n}(\omega)=\lambda \underline{X}_{n-1}(\omega)+\Delta t \mathcal{X}(n \Delta t) e^{-j \omega n \Delta t}$

for $\mathcal{X} \in \mathbb{R}^{m \times 1}$ defined as $\mathcal{X}(n \Delta t) \equiv \mathcal{X}_{n}=\left[\begin{array}{lll}x(n \Delta t) & u(n \Delta t)\end{array}\right]^{T}$, cf. (7). In (14), the vector $\underline{X}_{n}(\omega)$ is issued from:

$X^{T}=\left[\begin{array}{ccc}X_{1}\left(\omega_{1}\right) & \cdots & X_{1}\left(\omega_{M}\right) \\ \vdots & \vdots & \vdots \\ X_{m}\left(\omega_{1}\right) & \cdots & X_{m}\left(\omega_{M}\right)\end{array}\right]=\left[\underline{X}\left(\omega_{1}\right) \cdots \underline{X}\left(\omega_{M}\right)\right]$
As a result, denoting $\mathcal{E}_{n}^{\dagger}=\left[\begin{array}{lll}e^{j \omega_{1} n \Delta t} & \cdots & e^{j \omega_{M} n \Delta t}\end{array}\right]$, we can set the basis of a time-recursion as:

$X_{n}^{\dagger}=\lambda X_{n-1}^{\dagger}+\Delta t \mathcal{X}_{n} E_{n}^{\dagger}$, that yields (with $M_{n}=X_{n}^{\dagger} X_{n}$ ):

$M_{n}=\lambda^{2} M_{n-1}+\Delta t\left[M \Delta t \chi_{n} \chi_{n}^{T}+\lambda \chi_{n} E_{n}^{\dagger} X_{n-1}\right.$

$$
\left.+\lambda X_{n-1}^{\dagger} E_{n} X_{n}^{T}\right]=\lambda^{2} M_{n-1}+\sum_{i=1}^{3} B_{i} C_{i} D_{i}
$$

with: $\left\{\begin{array}{lll}B_{1}=\sqrt{M} \Delta t \mathcal{X}_{n} & C_{1}=1 & D_{1}=B_{1}^{T} \\ B_{2}=\Delta t \mathcal{X}_{n}=\sqrt{M} B_{1} & C_{2}=1 & D_{2}=\lambda \mathcal{E}_{n}^{\dagger} X_{n-1} \\ B_{3}=\lambda X_{n-1}^{\dagger} \mathcal{E}_{n}=D_{2}^{\dagger} & C_{3}=1 & D_{3}=\Delta t \mathcal{X}_{n}^{T}=B_{2}^{T}\end{array}\right.$

As $B_{1} \in \mathbb{R}^{m \times 1}$, we also benefit from:

$R_{n}=R\left(M_{n}\right)=\lambda^{2} I_{n-1}+B_{1} D_{1}+B_{2} R\left(D_{2}\right)+\left[B_{2} R\left(D_{2}\right)\right]^{T}$

Hence $M_{n}^{-1}$ (or $R_{n}^{-1}$ ) can be computed recursively by applying the matrix inversion lemma 3 times to the 3 additions of (16):

$(A+B C D)^{-1}=A^{-1}-A^{-1} B\left[D A^{-1} B+C^{-1}\right]^{-1} D A^{-1}$

with: $\left\{\begin{array}{l}A_{1}=\lambda^{2} M_{n-1} \\ A_{2}=\lambda^{2} M_{n-1}+B_{1} C_{1} D_{1}=A_{1}+B_{1} C_{1} D_{1} \\ A_{3}=\lambda^{2} M_{n-1}+B_{1} C_{1} D_{1}+B_{2} C_{2} D_{2}=A_{2}\end{array}\right.$ $A_{3}=\lambda^{2} M_{n-1}+B_{1} C_{1} D_{1}+B_{2} C_{2} D_{2}=A_{2}+B_{2} C_{2} D_{2}$

resulting in the following sequence of computation steps:

- $1 / A_{2}^{-1}=\left[A_{1}+B_{1} C_{1} D_{1}\right]^{-1}=\lambda^{-2} M_{n-1}^{-1}\left(I-\frac{B_{1} D_{1}}{s_{1}} M_{n-1}^{-1}\right)$

- 2/ $A_{3}^{-1}=\left[A_{2}+B_{2} C_{2} D_{2}\right]^{-1}=A_{2}^{-1}\left(I-\frac{B_{2} D_{2}}{s_{2}} A_{2}^{-1}\right)$

- 3/ $M_{n}^{-1}=\left[A_{3}+B_{3} C_{3} D_{3}\right]^{-1}=A_{3}^{-1}\left(I-\frac{B_{3} D_{3}}{s_{3}} A_{3}^{-1}\right)$

with $s_{1}=D_{1} M_{n-1}^{-1} B_{1}+\lambda^{2}, s_{2}=D_{2} A_{2}^{-1} B_{2}+1, s_{3}=D_{3} A_{3}^{-1} B_{3}+1$.

Finally, (17)-(21) permit the direct updating of $R_{n}^{-1}$ to be computed without requiring onboard a tricky matrix inversion, by using only a series of matrix additions and products. For the sake of simplicity, $\Sigma_{0}^{-1}$ was assumed to be 0 in the previous expression of $M_{n}$. Dealing with a priori knowledge is however possible by achieving at first a series of $m$ rank 1 corrections to $A_{1}^{-1}$ with the diagonal values of $\Sigma_{0}^{-1}$.

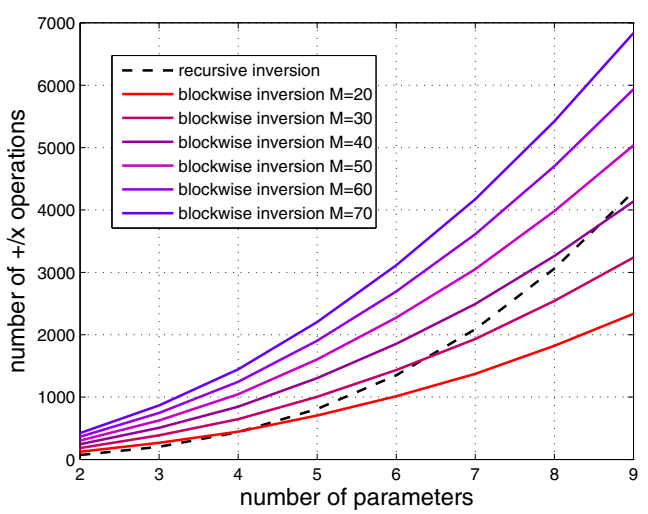

Figure 2: Computational costs of the recursive and blockwise inversions

Given that standard algorithms do not comply with certification and implementation constraints, another solution for computing $R_{n}^{-1}$ when $\operatorname{dim}\left(R_{n}\right)>3$ would consist in resorting to a blockwise inversion by iteratively partitioning $R_{n}$ into a series of submatrices of lower dimensions till analytical 
inverses can be got whenever dimensions 2 or 3 are reached. However, it is noteworthy that this approach requires the $R$ matrix to be computed in addition to the matrix products of the blockwise inverse. Fig. 2 compares (for different values of $m$ and $M$ ) the computational costs, expressed in terms of the number of floating-point operations, associated to the recursive algorithm (17)-(21) (including a priori knowledge) and to a blockwise inversion. For the usual values we are concerned with for on-line estimation ( $3 \leq m \leq 6, M \approx 50)$, the recursive algorithm proposed in this paper is clearly attractive: in the application case presented in §III, the cost is decreased more than twofold. Moreover, the blockwise algorithm would require the submatrices (and their Schur complement) to be invertible, a property that cannot be a priori guaranteed.

\section{RESULTS AND DISCUSSION}

In $\S I I I$, the recursive FD estimator described in $\S \mathrm{II}$ is used to develop an indirect adaptive control strategy in the framework of the EU-FP7 funded project RECONFIGURE (REconfiguration of CONtrol in Flight for Integral Global Upset REcovery). The goal of this project is to investigate and to evaluate aircraft guidance and control technologies that facilitate the automated handling of off-nominal/abnormal events, alleviate the pilot workload, and optimize the aircraft status by automatically reconfiguring the aircraft to an optimal flight condition (see http://reconfigure.deimos-space.com/). A detailed description of the benchmark model and of the fault/failure scenarios is given in $[9,17]$. The $V \& V$ process involves a nonlinear highly representative model of a generic AIRBUS civil aircraft. The benchmark contains a baseline gain scheduled PI controller, plus actuator and sensor models, measurement filters, as well as the control law protections.

During this project, an adaptive gain-scheduled flight control law has been designed off-line at first, by using a state-space modal technique to synthesize a LFT controller which would only depend on the major model aerodynamic parameters (see the companion paper [8]). The FD PID is implemented to track those time-varying parameters on-line, and to schedule the controller w.r.t. the provided estimates. In this paper, we only consider the longitudinal motions, and we focus on the $\mathrm{A} / \mathrm{C}$ lift, pitch and load factor equations that characterize the short-period dynamics. Hence, about a given flight condition, the stability derivatives to be tracked (physical parameters of the aerodynamic model) appear as pseudo-constants in a linearized state-space model (3)-type:

$\left\{\begin{array}{l}\dot{\alpha}=Z_{\alpha} \alpha+Z_{q} q+Z_{\delta} \delta_{m}+Z_{V} V \\ \dot{q}=M_{\alpha} \alpha+M_{q} q+M_{\delta} \delta_{m}+M_{V} V \\ N_{z}=N_{\alpha} \alpha+N_{q} q+N_{\delta} \delta_{m}+N_{V} V\end{array}\right.$

where the states $\alpha$ and $q$ are the angle of attack and the pitch rate, $V$ is the airspeed, $\delta_{m}$ is the elevator input, and $N_{z}$ is the load factor output. The influence of the altitude variations $(z)$ are neglected, as the aerodynamic coefficients only indirectly depend on $z$. Accordingly, (22) reduces to:

$\left[\begin{array}{ccc}\dot{\alpha} & \dot{q} & N z\end{array}\right]^{T}=\Theta\left[\begin{array}{ll}x_{\text {lon }}^{T} & u_{\text {lon }}^{T}\end{array}\right]^{T}$

where the $\Theta$ matrix includes the 12 parameters to be tracked and estimated, $x_{l o n}$ denotes the longitudinal states of interest and $u_{l o n}$ reduces to the elevator control $\delta_{m}$. In the context of the adaptive scheme, the main goal of the on-line estimator is to update the values of the five main stability derivatives $Z_{\alpha}, M_{\alpha}, M_{q}, M_{\delta}, N_{\alpha}$ used to schedule the controller [8], although the twelve coefficients of (22) have to be estimated.

To evaluate the proposed approach, the simulated scenario corresponds to a $380 \mathrm{sec}$. realistic flight profile including 4 successive stages (Fig. 3): a first steady flight at $(z=12500 \mathrm{ft}$, $\left.V_{c}=240 \mathrm{kts}\right)$, followed by a climb for $t \in[65,215]$ up to $20000 \mathrm{ft}$ and an acceleration for $t \in[215,300]$ up to $335 \mathrm{kts}$, and finally a new steady flight condition at $(z=20000 \mathrm{ft}$, $V_{c}=335 \mathrm{kts}$ ). The fault scenario which is considered is a loss of airspeed information; hence it is assumed that $V_{c}$ is no more available throughout the flight. The testing maneuvers selected by Airbus for the industrial validation of the controllers involve joystick doublet-type signals. So, to check the performances of the adaptive process, usual stick inputs are applied to the $N_{z c}$ set points (in blue) during the $1^{\text {st }}$ and $4^{\text {th }}$ parts of the flight with autopilot and auto-throttle switched off. The aircraft mass is $260 \mathrm{t}$ and the CoG is located at $28 \%$ (of the mean chord).
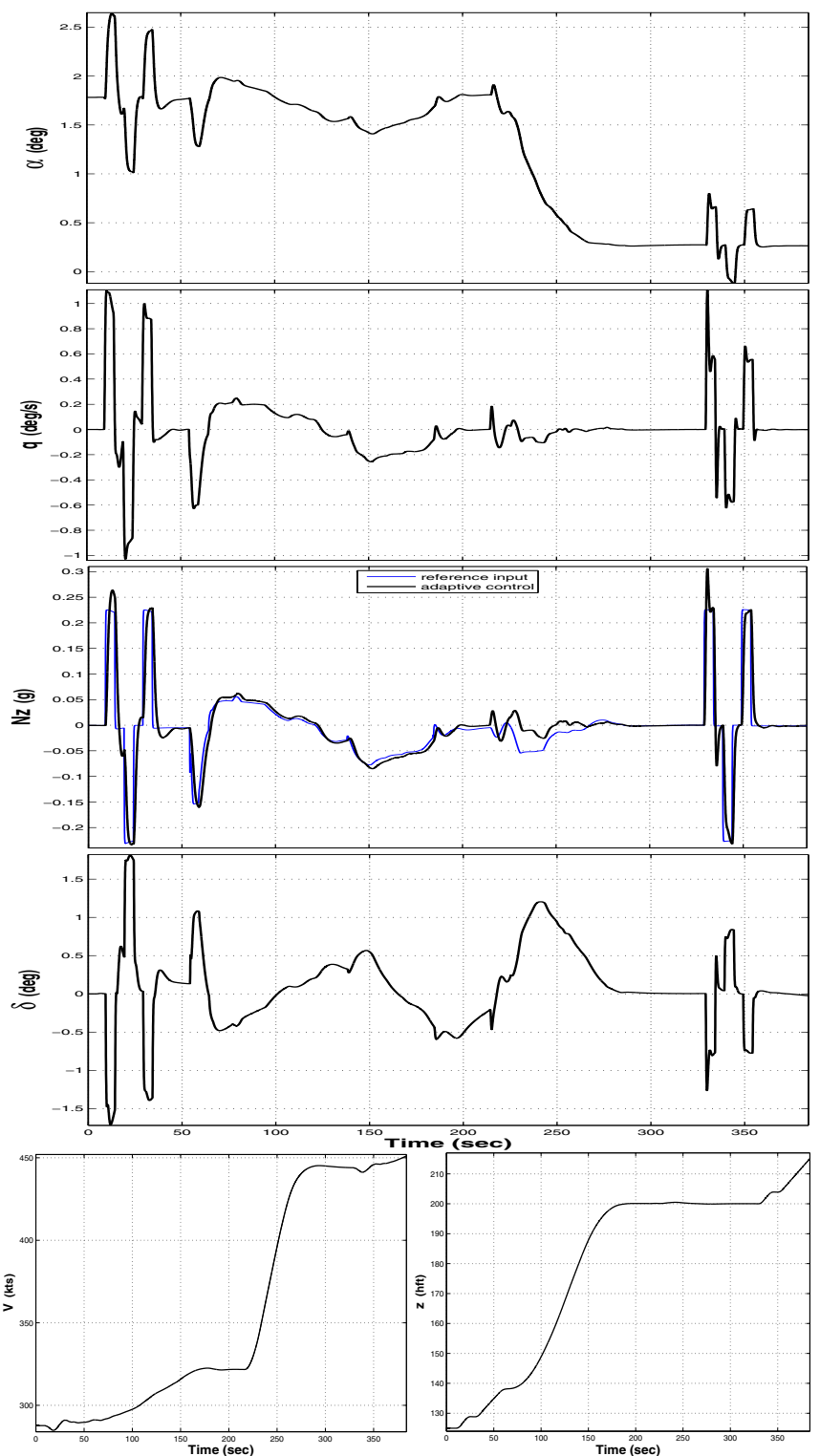

Figure 3: Time history of the longitudinal aircraft flight parameters 
Regarding the tuning of the FD Algorithm (FDA) described in $\S$ II, the frequency band chosen for the FT computations comprises $M=43$ frequency samples equally spaced in the range $0.1-0.52 \mathrm{~Hz}$ with a frequency resolution of $0.01 \mathrm{~Hz}$, to include the rigid-body modes of the airplane and to filter out low frequency phenomena. It is worth noting that the spacing does not need to be uniform for this FDA since no standard FFT is used. All the data are high-pass filtered prior to performing FT to comply with the assumption of linearization about a trim condition, and to filter out low frequencies. A $1^{\text {st }}$ order filtering is implemented with a time constant of $3 \mathrm{sec}$. After encoding the algorithm with operators compliant to the SCADE software (Safety Critical Application Development Environment), the resulting computational burden evaluated by Airbus is about $1.5 \mathrm{~ms}$, which is acceptable and could be further improved by updating the estimates at a lower rate.

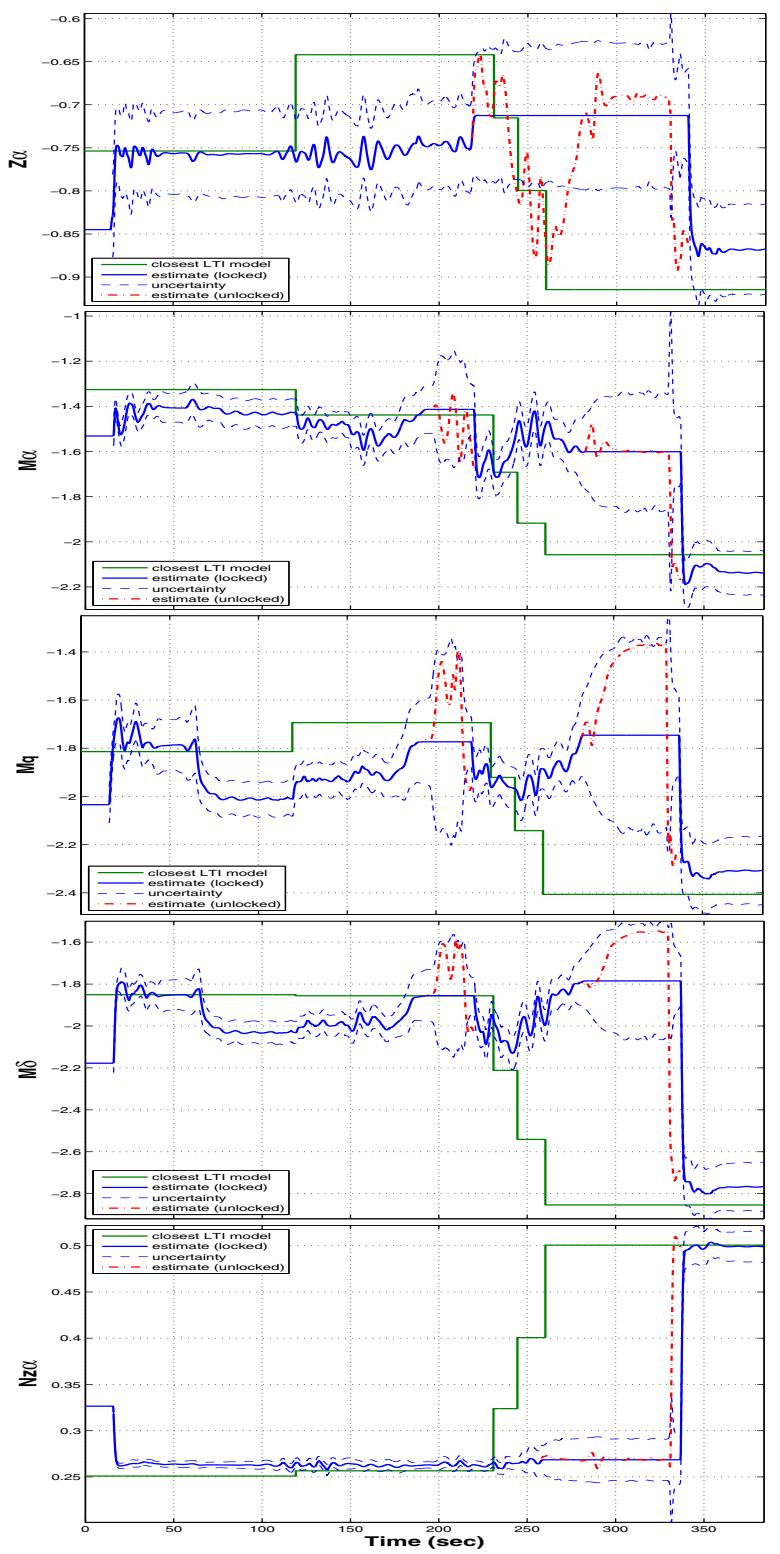

Figure 4: Time history of the estimated parameters

The results achieved by the FDA are displayed with solid blue lines in Fig. 4, that only plot the 5 (out of 12) coefficients required for the adaptive control. The parameter uncertainties $(3 \sigma)$ are plotted in blue dashed lines and are used to freeze the estimates whenever they are too high (straight lines in the plots). While the estimates are invalid, the last valid estimated value is used to schedule the controller as long as the estimates are not declared reliable again. The green curves give an idea of the reference values drawn from the closest LTI model (just one of 25 for this mass/CoG). Due to a loose mesh of the models in the flight domain, these values are only plotted as a rough guide, and this must be kept in mind when judging the estimates (the true linearized values being unavailable).

Owing to the simulated scenario, the variations that should be tracked result either from a change in the flight condition or, at the start of the run, from a misknowledge of the initial parameter values. Both of them demonstrate the interest of the adaptation to cope with modeling uncertainties. It is worth noting that the continuous variation of $V / z$ is monitored by the estimation algorithm during level changes, and that the parameters accuracy can also be used to trigger a freeze of the estimates whenever the uncertainty becomes too high (not enough excitation or rapid changes in the flight condition). Hence, 2 options are available to manage the FDA results, and to feed the adaptive controller in case of ill-conditioning: - to proceed with a continuous estimation while reinforcing the regularization temporarily using a priori knowledge; $\boldsymbol{\square}$ to lock the parameters to their last estimated values as long as they are unreliable.

In addition, the forgetting mechanism of (13) can be activated during level changes to speed up the convergence of the parameters to their new steady values, or the FDA can be automatically reset as soon as the new flight condition is reached. Both strategies have their own pros and cons. The former requires the value of the forgetting factors to be tuned (which could depend on the rate of changes), but permits the new estimates to be updated as soon as some excitation is available about the new flight condition. The latter avoids any tuning and relies on frozen values during the dead zone, but slows down the updating about the new flight condition due to a transient phase after resetting the FDA. It is noteworthy that all these possibilities (reinforced regularization/locked parameters, forgetting/resetting) have been evaluated but cannot be displayed by lack of space; they result only in very slight differences in the performances plotted in Fig. 3 .

The point is that, thanks to a simple monitoring of the changes in flight condition and of the parameter uncertainty provided by the FDA itself, different strategies can be temporarily triggered resulting in different settings of the PID process: to activate the forgetting mechanism by switching to a $\lambda<1$, to strengthen the regularization by decreasing some components of $\Sigma_{0}$, to freeze the values of the estimates delivered to the controller, and possibly to reset the procedure. On the other hand, owing to the properties of the recursive FT, it should be understood that the information provided by the data continuously accumulates and contributes to improve the parameter accuracy. Consequently, if excitation is lacking for a while, no parameter divergence is to be feared, except in case older information is progressively forgotten. That is why forgetting should not be permanently activated, and why the estimation accuracy is so valuable and should also be monitored to reconfigure the FDA whenever necessary. 
For example, in Fig. 4 the forgetting mechanism is (only) triggered when significant $z / V$ changes are detected providing a way to adapt more rapidly to varying conditions. The results corresponding to the 2 previous options are illustrated: in blue color when the parameters are locked in case of unreliable estimates, in red dashdot lines when the regularization is reinforced instead. It should be pointed out that the latter was not used during the closed-loop simulation of Fig. 3, but is still plotted in Fig. 4 to evaluate the behavior of the estimates, should this option be used. However, regardless of the selected option, the closed loop responses of Fig. 3 are almost the same: the estimates are typically unreliable when closed loop excitation is poor, so that the gap between the performances is insignificant.

It should also be pointed out that with such a poor excitation (see $N_{z c}$ in Fig. 3 during the $2^{\text {nd }} / 3^{\text {rd }}$ stages), some parameters can't be estimated properly. Hence to prevent from correlation issues or ill-conditioning, a tuned-down modeling or some regularization is required. Here, a priori knowledge via the Bayesian formulation of (7) is introduced, w.r.t. the values of the damping coefficients $Z_{q}$ (trickier to estimate even with proper excitation), and mostly of the speed derivatives $C_{V}$ (the speed variations about the trim condition are very small). When the regularization needs to be reinforced, the variation of the pitching derivatives is also overweighted, especially to avoid data collinearity between $M_{q}$ and $M_{\delta}$.

The plots of Fig. 4 deserve some comment. During the $3^{\text {rd }}$ part of the scenario, for $t \in[200,330]$, the excitation level is very low (remember that the data of Fig. 3 are high-pass filtered before being processed by the FDA), and hence the parameter inaccuracy results either in freezing or in strongly regularizing the estimation process (blue/red curves). This process is also not exactly the same for the different parameters as the lift, pitching and load factor equations are processed separately. Hence, the trends indicated by the (green) reference values cannot be mimicked by the estimates, and instead they are drawn towards the prior valid estimates or towards average reference values used as a priori knowledge (e.g., mean values for a given altitude). As soon as sufficient excitation is available again, and the accuracy is good enough (time to store information through the recursive updates of the FT), the estimates are freed again and converge rapidly to suitable values.

In steady state conditions, the estimates provided by the FDA compare very favorably to their reference values. Thanks to the noise filtering outside the selected bandwidth, the FDA does not require much time to accumulate enough information and to recover proper estimates. In case of continuous estimation, the convergence delay is even shorter as the estimates may be reliable enough although their standard errors are not fully satisfying.

As regards the performance of the adaptive controller, we have to refer to the plots of the load factors in Figs. 3 and 5 ( $N_{z}$ vs $N_{z c}$ ). Fig. 5 focuses on the responses during the first and last $40 \mathrm{~s}$ of the flight. The blue line corresponds to the $N_{z c}$ stick input settings, and the black one to the $N_{z}$ signals. As soon as new reliable estimates are available for the scheduling, much better performances are recovered thanks to the adaptive process resulting in proper overshoot and settling time (please refer to the companion paper [8] for a comprehensive description of the control aspects). Hence in faulty situations [9], the proposed strategy can avoid switching to a more robust but less efficient control law (e.g., when the airspeed data is missing for the usual scheduling process due to sensor faults).
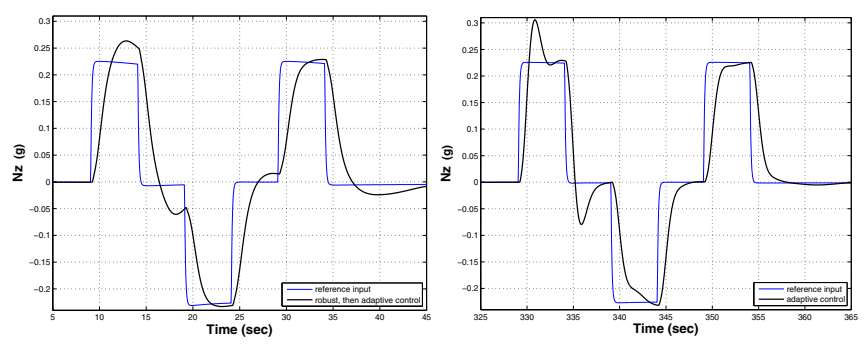

Figure 5: Zooms in on the load factor responses

\section{CONCLUSIONS AND PROSPECTS}

A fully recursive estimation scheme was developed in the frequency domain to track time-varying parameters, more likely to comply with the requirements of embedded $\mathrm{A} / \mathrm{C}$ implementation than the standard existing algorithms. Some issues related to the computation of state derivatives are also properly settled to get recursive expressions of the Fourier Transforms. This recursive estimation algorithm is part of an indirect adaptation process, permitting an LFT flight controller to be designed and to be scheduled w.r.t. the estimated model coefficients when the airspeed information is missing. Nonlinear simulations, achieved via the industrial benchmark of the RECONFIGURE project, show the capability to control the airplane along a trajectory, a more difficult problem than controlling about a steady flight point since the time-varying parameters have to be estimated despite poor closed loop excitation. The computational burden and complexity of the global adaptive scheme are also compliant with an embedded implementation on existing computers.

These results are very promising as regards a possible application to civil aircraft, but of course further industrial validation would be needed using extensive sets of simulations including a wide range of operational conditions (realistic flight scenarios, external disturbances, pilot maneuvers, and so on). Tools like the Functional Engineering Simulator, implemented during the RECONFIGURE project to achieve a traditional Monte Carlo analysis as a preliminary step of the industrial V\&V process [17], should be extended to be suited to this need. Actually, to evaluate the proposed adaptive approach, longer operational scenarios are required to benefit from on-line parameter estimation, including changes in the flight conditions and unexpected faults occurring during the flight. A transient stage at the start of the simulation is also required to initialize the algorithm and to accumulate some information prior to delivering reliable estimates to the gainscheduling process. These constraints spring from the fact that the adaptive controller is not pre-computed once for all, and hence is not available at the initial starting time.

To contribute to the $\mathrm{V} \& \mathrm{~V}$ process, avenues do exist for such complex controllers; for instance, a practical approach for the worst-case validation of a similar adaptive scheme 
was already investigated by Airbus in [28]. On the other hand, this validation involves integrated estimation and control components, but some theoretical guarantees regarding the robustness to transient or asymptotic estimation errors can be proved at first using $\mu$ and IQC analysis techniques [8]. Stability and performance properties of the adaptive closed loop can also be enforced thanks to more elaborated strategies, e.g. by switching to a robust controller in some transient situations. More details are available in the companion paper [8].

\section{REFERENCES}

[1] Astrom K.J., Wittenmark B., Adaptive Control, $2^{\text {nd }}$ edition, AddisonWesley, Reading, 1995.

[2] Basappa, Jategaonkar R.V., "Evaluation of recursive methods for aircraft parameter estimation", AIAA AFM, Providence, USA, 2004.

[3] Buffington J. et al., "Integration of on-line system identification and optimization-based control allocation", AIAA GNC, Boston, 1998.

[4] Chandler P.R., Pachter M., Mears M., "System identification for adaptive and reconfigurable control", JGCD, 18(3):516-524, 1995.

[5] Ducard G., Geering H.P., "A reconfigurable flight control system based on the EMMAE method", ACC, Minneapolis, USA, 2006.

[6] Ferreres G., Antoinette P., "Robust gain scheduling techniques for adaptive control", ECC, Budapest, Hungary, 2009.

[7] Ferreres G., Antoinette P., "Adaptive control using robustness and gain-scheduling", Journal Européen des Systèmes Automatisés, 45(4-6):279-298, 2011.

[8] Ferreres G., Hardier G., Seren C., "Adaptive control of a civil aircraft through on-line parameter estimation", Systol'16, Barcelona, Spain, 2016.

[9] Goupil P. et al., "AIRBUS efforts towards advanced real-time fault diagnosis and fault tolerant control". $19^{\text {th }}$ IFAC World Congress, Cape Town, South Africa, 2014.

[10] Gustafsson F., Adaptive Filtering and Change Detection, John Wiley and Sons Ltd, 2000.

[11] Han Y., Kim Y., Kim H., "Frequency and time domain online parameter estimation for reconfigurable flight control system", AIAA Infotech Aerospace, Seattle, USA, 2009.

[12] Hanlon P.D., Maybeck P.S., "Multiple-model adaptive estimation using a residual correlation KF bank", IEEE Trans. on aerospace and electronic systems, 36(2):393-406, 2000.

[13] Hardier G., Bucharles A., "On-line parameter identification for inflight aircraft monitoring", 27 $7^{\text {th }}$ ICAS Congress, Nice, France, 2010

[14] Hardier G., "An extended U-D algorithm with multiple forgetting factors for RLS estimation of model parameters", Safeprocess, Paris, France, 2015.

[15] Jategaonkar R.V., Flight vehicle system identification: a time domain methodology, AIAA editions, Vol. 216, Reston, USA, 2006.

[16] Jiang J., Zhang Y., "A revisit to block and recursive least squares for parameter estimation”, Computers \& Electrical Engineering, 30, 2004.

[17] Kerr M.L. et al., "RECONFIGURE FP7 project: preliminary results and contributions", Systol'16, Barcelona, Spain, 2016.

[18] Klein V., Morelli E., Aircraft system identification: theory and practice, AIAA Education Series, Reston, 2006.

[19] Larsson R., Enqvist M., "Sequential aerodynamic model parameter identification". 16 $6^{\text {th }}$ IFAC SYSID, Brussels, Belgium, 2012.

[20] Ljung L., "Asymptotic behaviour of the extended KF as a parameter estimator for linear systems", IEEE Trans. on Automatic Control, 24(1):36-50, 1979.

[21] Ljung L., Soderstrom T., Theory and practice of recursive identification, MIT Press, Cambridge, 1983.

[22] Ljung L., Gunnarsson S., "Adaptation and tracking in system identification: a survey", Automatica, 26(1):7-21, 1990.

[23] Melody J.W. et al., "Parameter identification for in-flight detection and characterization of aircraft icing", CEP, (8):985-1001, 2000.

[24] Morelli E., "Real-time parameter estimation in the frequency domain", $J G C D, 23(5), 2000$.

[25] Morelli E., "Practical aspects of the EE method for aircraft parameter estimation", AIAA AFM, no 2006-6144, Keystone, USA, 2006.
[26] Morelli E., Smith M.S., "Real-time dynamic modeling - Data information requirements and flight test results", Journal of Aircraft, 46(6):1894-1905, 2009.

[27] Oudin S. et al., "Adaptive LFT control of a transport aircraft on the lateral axis", IEEE MSC, Dubrovnik, Croatia, 2012.

[28] Oudin S. et al., "Design and worst-case validation of a longitudinal adaptive flight control law: a practical approach", AIAA GNC, Boston, USA, 2013.

[29] Smith L. et al., "Regularization techniques for real-time identification of aircraft parameters", AIAA GNC, New Orleans, USA, 1997.

[30] Smith M.S. et al., "Real-Time Stability and Control Derivative Extraction from F-15 Flight Data”, NASA TM-2003-212027, 2003.

[31] Tang L. et al., "Methodologies for adaptive flight envelope estimation and protection", AIAA AFM, no 2009-6260, Chicago, USA, 2009. 